\title{
Real-World Treatment Costs and Care Utilization in Patients with Major Depressive Disorder With and Without Psychiatric Comorbidities in Specialist Mental Healthcare
}

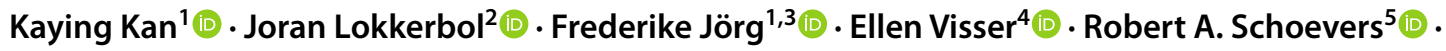 \\ Talitha L. Feenstra ${ }^{6,7}$ (D)
}

Accepted: 18 February 2021 / Published online: 16 March 2021

(c) The Author(s) 2021

\begin{abstract}
Background The majority of patients with major depressive disorder (MDD) have comorbid mental conditions.

Objectives Since most cost-of-illness studies correct for comorbidity, this study focuses on mental healthcare utilization and treatment costs in patients with MDD including psychiatric comorbidities in specialist mental healthcare, particularly patients with a comorbid personality disorder (PD).

Methods The Psychiatric Case Register North Netherlands contains administrative data of specialist mental healthcare providers. Treatment episodes were identified from uninterrupted healthcare use. Costs were calculated by multiplying care utilization with unit prices (price level year: 2018). Using generalized linear models, cost drivers were investigated for the entire cohort.

Results A total of 34,713 patients had MDD as a primary diagnosis over the period 2000-2012. The number of patients with psychiatric comorbidities was 24,888 (71.7\%), including 13,798 with PD. Costs were highly skewed, with an average \pm standard deviation cost per treatment episode of $€ 21,186 \pm 74,192$ (median $€ 2320$ ). Major cost drivers were inpatient days and daycare days (50 and $28 \%$ of total costs), occurring in 12.7 and $12.5 \%$ of episodes, respectively. Compared with patients with MDD only $(€ 11,612)$, costs of patients with additional PD and with or without other comorbidities were, respectively, $2.71(p<.001)$ and $2.06(p<.001)$ times higher and were $1.36(p<.001)$ times higher in patients with MDD and comorbidities other than PD. Other cost drivers were age, calendar year, and first episodes.

Conclusions Psychiatric comorbidities (especially PD) in addition to age and first episodes drive costs in patients with MDD. Knowledge of cost drivers may help in the development of future stratified disease management programs.
\end{abstract}

\section{Introduction}

Major depressive disorder (MDD) is a highly prevalent mental disorder, with more than 264 million people affected worldwide in 2017 [1]. In the Netherlands, the lifetime prevalence of MDD is estimated at around $18.7 \%$ with a 12 -month prevalence of 5.2\% [2]. This is comparable to the prevalence in other high-income countries (14.6 and 5.5\%, respectively) [3]. MDD has a major impact on patients' lives and is associated with limitations in multiple dimensions of functioning, both physical and social. Those limitations are comparable to or worse than those of patients with other chronic medical

Kaying Kan

K.Kan@umcg.nl

Extended author information available on the last page of the article conditions such as arthritis and diabetes [4]. In addition, it involves considerable loss in productivity and work cut-back [5]. Hence, MDD is a large burden for society, remaining one of the leading causes of years lived with disability [1] and total disability-adjusted life-years globally $[6,7]$. It is expected to be among the top three diseases with the highest global burden of disease in 2030 [8].

MDD often has a chronic-intermittent course [9]. In specialist mental healthcare settings, where patients are more severely depressed, recurrence rates are high; after 5 years, $60 \%$ of patients have experienced a recurrent depression, and this rate increases to $85 \%$ after 15 years [10]. Around $57 \%$ of patients diagnosed with depression or anxiety who recently visited their general practitioner (GP) received professional treatment in the general practice setting or the mental healthcare setting [11]. Currently, depression is one of the most 


\section{Key Points for Decision Makers}

In specialist mental healthcare, major depressive disorder is highly comorbid with personality disorder and other psychiatric comorbidities.

Health service use and related treatment costs are significantly higher in patients with major depressive disorder and a comorbid personality disorder than in patients with major depressive disorder alone in specialist mental healthcare.

The results regarding cost drivers of treatment, accounting for the presence of comorbidity, provide the opportunity for future stratified disease management programs.

For example, patients at risk for burdensome trajectories likely benefit from intensive treatment strategies tackling several disorders at the same time.

expensive diseases in the Netherlands, with an expenditure of almost $€ 1.1$ billion annually (at least $1.3 \%$ of the total expenditure on health and welfare) [12].

Previous research has shown that patients with MDD often have other mental disorders $[13,14]$. The course of illness for patients with MDD and coexisting mental or physical illnesses is less favorable. They have more severe symptoms, lower levels of functioning, and less recovery than patients with MDD alone [15]. The prevalence of a comorbid personality disorder (PD) in patients with MDD is high at $45 \%$ and rises to $60 \%$ in patients with dysthymic disorders [16]. Although the majority of studies demonstrated that a comorbid PD with MDD was associated with poorer outcomes for depression than MDD alone [17-19], other studies found no differences (at long-term follow-up) [19, 20] or did find a negative effect on depression outcome but not on functioning or quality-of-life outcomes [21]. Looking at healthcare utilization, patients with PD had more psychiatric inpatient, outpatient, and pharmacological treatment than patients with MDD as a single diagnosis [22]. Additionally, patients with MDD and comorbid PD were more likely to have hospitalizations, higher rates of recurrent depressions, longer mean length of stay [23], and longer duration of depressive episodes than patients with MDD without comorbid PD [24].

Several studies have investigated the direct and indirect costs of MDD from a societal perspective [25-30]. The healthcare costs associated with MDD in primary care patients have also been a focus of research [31, 32]. Several cost-of-illness studies evaluated MDD with comorbid somatic disorders $[33,34]$ or allowed for MDD-related treatment/costs [35]. At the same time, these studies included relatively short follow-up times of 1-2 years. One study demonstrated that the overall effect of neuroticism, a personality characteristic, on the use of somatic and mental healthcare was associated with enormous economic costs, exceeding those of common mental disorders, including mood disorders [36]. To our knowledge, no studies have investigated in detail the treatment costs of patients with MDD and other psychiatric comorbidities in the specialist mental healthcare setting in unselected observational data with a large follow-up time. The impact of various psychiatric comorbidities, including PD, on healthcare utilization and treatment costs remain underexposed.

The aims of our study were to (1) compare specialist mental healthcare utilization and related costs of treatment episodes in patients diagnosed with MDD with and without psychiatric comorbidities, with a particular focus on comorbid PD, and (2) investigate which patient characteristics and clinical variables were driving treatment costs.

\section{Methods}

\subsection{Data Source}

For this retrospective observational study, we used a large administrative mental healthcare database: Psychiatric Case Register North Netherlands (PCRNN). This register contained patient-level specialist mental health service use and clinical diagnosis data for patients in the northern region of the Netherlands (1.7 million inhabitants) between 1 January 2000 and 31 December 2012. In the PCRNN, data were available for the majority of specialist mental healthcare organizations (approximately $75 \%$ ) providing care to patients with moderate to severe mental health disorders. The PCRNN data consisted of patient demographics, main and secondary diagnoses according to the Diagnostic and Statistical Manual of Mental Disorders (DSM) [37], and the date and type of mental health service use. Usage of mental health services was divided into five categories: (1) inpatient day (24-h treatment), (2) day treatment, (3) psychiatric home care service, (4) community-based daycare, and (5) outpatient visit.

\subsection{Study Population}

We included patients registered with unipolar MDD as primary diagnosis and who had at least one record of mental health service use in the PCRNN. We included the following DSM-IV diagnosis codes: 296.2-296.36 [37]. We distinguished between patients with and without a comorbid PD (DSM-IV codes 301.0-301.9) diagnosed in the cohort period of the study. We also distinguished between the presence or absence of other psychiatric comorbidities, including 
anxiety disorders, bipolar disorders, schizophrenia disorders, substance use disorders, and a category "other," covering the remainder of disorders. For more information regarding the definitions of the psychiatric comorbidities, see the electronic supplementary material (ESM)-A. We distinguished four groups of patients: (1) patients with MDD only, (2) patients with MDD and other psychiatric comorbidities other than PD, (3) patients with MDD and comorbid PD without other psychiatric comorbidities, and (4) patients with MDD and other psychiatric comorbidities, including PD.

\subsection{Defining Treatment Episodes}

Mental healthcare costs were estimated per treatment episode. The first treatment episode started with the first mental health service use record after the date of MDD diagnosis as primary diagnosis. The end of a treatment episode was the last mental health service use record after which no mental health service contacts were registered for 6 consecutive months. The first mental health service use contact after 6 consecutive months with no mental health service use contacts marked the start of a subsequent treatment episode. From 1 January 2013, the PCRNN database ceased to exist in that form, therefore no data were available after 31 December 2012. Treatment episodes that started after 1 July 2012 were considered censored episodes. These episodes were excluded from the analysis as they may have resulted in higher costs if they continued in 2013. The end date of each patient's last follow-up was determined when no mental health service contacts were registered for 6 consecutive months within the cohort period.

A first treatment episode could be distinguished as an episode without any mental health service use in the cohort period prior to MDD diagnosis and an episode with previous mental health service use for treatment not related to MDD in the cohort period.

\subsection{Outcome Measures}

Outcomes of interest were average and median costs of treatment episodes in patients with MDD as primary diagnosis with and without other psychiatric comorbidities. Subsequently, drivers of treatment costs (patient characteristics [sex, age] and clinical variables [presence of psychiatric comorbidities, including PD, and first or subsequent treatment episode] were investigated. Sex, age, MDD diagnosis, and first or subsequent episode were measured prior to the treatment episode. The presence of psychiatric comorbidities was measured either at baseline or during an episode. Frequency counts and percentages were used to summarize categorical variables. We reported both means and medians for continuous variables because costs and episode duration were highly skewed. Differences in costs between these four groups of patients were compared.

\subsection{Data Analytic Procedures}

Patient characteristics, mental health service use, treatment episode duration, and treatment costs per treatment episode were summarized descriptively. Treatment costs in specialist mental healthcare did not include treatment costs related to other non-mental-health-related chronic conditions. In this study, we used the healthcare perspective, applying unit prices of the five health service use categories to calculate the direct costs of treatment [38]. The five types of health service use were valued using unit costs per category and summed per treatment episode. Unit prices were taken from the Dutch Costing Manual of the National Health Care Institute [38] and indexed at the price level of year 2018. Comparisons of group characteristics were analyzed using either analysis of variance or generalized linear model (GLM) for continuous outcomes, Kruskal-Wallis test or Mann-Whitney test for non-normally distributed continuous or ordinal outcomes for two or more groups, or the chi-squared $\left(\chi^{2}\right)$ test for categorical outcomes. Within the group comparisons, a Bonferroni correction was applied to correct for multiple testing.

A GLM with a gamma distribution and a log-link function was used to assess the cost drivers of treatment in the PCRNN cohort. In the analyses, we additionally corrected for treatment episode duration, as cost of treatment will depend on duration (together with intensity) of treatment. The GLM permits flexible modeling of covariates and is recommended for right-skewed, non-normally distributed data, which usually applies to cost data [39]. Models were compared using the Akaike information criterion (AIC), and the best fitting model is presented.

Depending on the type of test, a $p$-value of $<0.05$ or the Bonferroni-corrected $p$-value determined statistical significance. All analyses were conducted using STATA/SE version 16.0 (StataCorp LLC, College Station, TX, USA).

\subsection{Sensitivity Analyses}

Sensitivity analyses were performed on the definition of treatment episodes by varying the time gap allowed between mental health service contact moments. In the sensitivity analyses, the start of a treatment episode was defined similarly, i.e., as the first mental health service contact after the date of MDD as a primary diagnosis. We defined the treatment episode as having ended when no mental health service use contacts were registered for 3 and 9 consecutive months, respectively. After this period, the first consecutive mental health service use record was considered the start of a subsequent episode, and so on. 


\section{Results}

\subsection{Study Population}

The demographic and clinical characteristics of the study sample are shown in Table 1. Of the 34,713 patients, $61.8 \%$ were female, and the average age was $44.3 \pm 18.3$ years at first MDD diagnosis in the specified cohort period. Almost two-thirds of the patients diagnosed with MDD had one treatment episode in specialist mental healthcare, and over one-third of the patients had one or more subsequent treatment episodes during the cohort period. After the removal of nine patients with missing sex data and the 2420 censored episodes, 52,667 treatment episodes remained. There were no missing values for the other variables in our data. Over $70 \%$ of the patients had psychiatric comorbidities, and $39.7 \%$ of the patients in the entire cohort were diagnosed with a comorbid PD.

Table 1 Demographic and clinical characteristics of the study sample

\begin{tabular}{ll}
\hline Variable & $N=34,713$ \\
\hline Sex, \% female & 61.8 \\
Age at the time of first MDD diagnosis, \% of total & \\
sample & \\
$<18$ & 5.1 \\
$18-65$ & 80.5 \\
$>65$ & 14.4 \\
Mean, years & $44.3 \pm 18.3$ \\
Number of treatment episodes after MDD diagnosis, & \\
\% of total sample & \\
One & 65.0 \\
Two or more & 35.0 \\
Range & $1-10$ \\
Comorbid personality disorder, \% yes & 39.7 \\
Number of psychiatric comorbidities (including per- & \\
sonality disorder) & \\
MDD only & 9825 \\
One additional diagnosis & 12,504 \\
Two additional diagnoses & 8292 \\
Three additional diagnoses & 3479 \\
Four additional diagnoses & 552 \\
Five additional diagnoses & 57 \\
Six additional diagnoses & 4 \\
Follow-up time in the cohort, days & \\
Mean & \\
Median & 773 \\
\hline
\end{tabular}

Data are presented as \% or mean \pm standard deviation unless otherwise noted

$M D D$ major depressive disorder

\subsection{Costs of First Versus Subsequent Treatment Episodes}

Table 2 shows the costs for first and subsequent treatment episodes for the entire group of patients diagnosed with MDD with or without any comorbidities. The average cost of a treatment episode was $€ 21,186 \pm 74,192$, with a median cost of $€ 2320$ (not shown in Table 2). Comparing the differences in mean costs between the three types of episodes using a Kruskal-Wallis H test, as well as a GLM with gamma distribution and log-link function, showed that the mean costs of the three types of treatment episodes differed significantly. First episodes of patients with a history of mental health service use for a non-MDD-related psychiatric diagnosis were 0.74 times cheaper $(p<.001 ; 95 \%$ confidence interval [CI] 0.68-0.81) than the first episodes of patients without a history of mental health service use for a non-MDD-related psychiatric diagnosis. Mean costs of subsequent episodes were 0.43 times cheaper $(p<.001 ; 95 \%$ CI 0.40-0.46) than the first episodes of patients without a history of mental health service use for a non-MDD-related psychiatric diagnosis.

The average duration of treatment for the entire study sample was 579 days (19 months), with a median duration of 308 days (10 months). In $25 \%$ of the cases, the treatment episodes lasted longer than 700 days. The duration of a treatment episode differed significantly between the three types of episodes $\left(F(2,52,664)=989.24, p<.001\right.$ and $\chi^{2}(2)=$ $2894, p<.001$ ), with the longest duration for a first treatment episode in patients with no history of mental health service use and shortest in a subsequent episode.

\subsection{Patient Characteristics, Mental Health Service Use, and Treatment Costs of Patients with Major Depressive Disorder With and Without Psychiatric Comorbidities}

Table 3 presents the patient characteristics, mental health service use, and treatment costs of the four patient groups defined in Sect. 2.2. Major cost drivers for the entire cohort were inpatient days and daycare days (50 and $28 \%$ of total costs). These occurred in 12.7 and $12.5 \%$ of episodes, respectively (not shown in Table 3). Patients' sex differed significantly in groups 1 and $2\left(\chi^{2}(1)=24.86, p<.001\right)$, groups 2 and $4\left(\chi^{2}(1)=13.53, p<.001\right)$, and groups 2 and $3\left(\chi^{2}(1)=17.49, p<.001\right)$. A statistically significant difference between the underlying distributions of age was found in all groups of patients $(F(3,34,709)=441.58, p<.001)$.

The number of subsequent treatment episodes differed significantly between the four groups $\left(\chi^{2}(3)=3700, p<\right.$ .001). Subsequent treatment episodes occurred the least in group 1 and were most frequent in group 4 . Furthermore, the four groups differed significantly in the amount of mental 
Table 2 Costs of treatment episodes

\begin{tabular}{llll}
\hline & First treatment episodes & & $\begin{array}{l}\text { Subsequent treatment } \\
\text { episodes }\end{array}$ \\
\cline { 2 - 4 } & No history of mental health service use & History of mental health service use & 17,954 \\
$\mathrm{~N}$ & 25,976 & 8737 & $11.9 \pm 43.7$ \\
Treatment costs per episode, $€ 1000$ & & $20.7 \pm 65.7$ & 1.3 \\
Mean & $27.8 \pm 91.0$ & 2.6 & $376 \pm 509$ \\
Median & 3.1 & $626 \pm 758$ & 202 \\
Treatment duration, days & & 350 & $<.001^{*}$ \\
Mean & $704 \pm 905$ & 372 &
\end{tabular}

Data are presented as mean \pm standard deviation or median

*Significant $p$-value for all subgroups

health service contacts per treatment episode $\left(\chi^{2}(3)=1839\right.$, $p<.001)$, treatment episode costs $\left(\chi^{2}(3)=1778, p=.0001\right)$, and treatment durations $\left(\chi^{2}(3)=1617, p<.001\right)$. Treatment episode duration was shortest in groups 1 and 2 and longest in groups 3 and 4 . Median costs of treatment episodes increased from $€ 1508$ in group 1 to $€ 3480$ in group 4. Patients with a comorbid PD (groups 3 and 4) more often used different types of mental health services than patients with MDD without PD (groups 1 and 2).

\subsection{Relation Between Patient and Clinical Characteristics and Treatment Costs}

Table 4 presents the results of the GLM with a log-link and gamma distribution. The GLM model was selected based on the best AIC and log-likelihood statistic (see ESM-B). Higher age, the presence of psychiatric comorbidities, year of start treatment episode, and a first treatment episode after MDD diagnosis were significantly associated with higher treatment costs. A subsequent treatment episode was negatively associated with treatment costs.

\subsection{Sensitivity Analyses: Varying the Definition of Treatment Episodes}

Table 5 presents the results of the sensitivity analyses. Using a treatment episode definition of 3 consecutive months of no mental health service use, the median duration of a treatment episode (167 days) was lower than the definition of 6 months (308 days) or 9 months (371 days). In the sensitivity analysis using a treatment episode definition of 9 consecutive months of no mental health service use, mean and median costs of a treatment episode approached the 6-month definition (mean $\pm € 22,500$, median $\pm € 2550$ ).

The variables that were consistently (significant or insignificant in all sensitivity analyses) associated with treatment costs were age, patient group (group 1-4), year of treatment episode start, and whether the treatment episode was a first or subsequent episode. In the sensitivity analyses, sex was not consistently associated with treatment costs.

\section{Discussion}

This observational cohort study showed that the treatment of patients with MDD and psychiatric comorbidities in specialist mental healthcare involved more health service use and higher costs than treatment of patients with MDD only. In particular, a comorbid PD resulted in more health service use and costs than other psychiatric comorbidities. These findings underscore the importance of disease management programs targeting patients with a combination of disorders. For the entire group of patients, a first treatment episode was significantly and robustly more costly than a subsequent treatment episode. Higher age, coexisting psychiatric disorders including PD, and year of treatment start were significantly associated with higher treatment costs.

We observed a median treatment duration of 10 months, and almost one-quarter (23\%) of the study population remained in treatment after 2 years. Previous epidemiological studies found depressive episode durations of 3-12 months [40, 41]. Spijker et al. [41] found that approximately $20 \%$ of patients sampled from the general population had not recovered in 24 months. Our results seem comparable.

In our study sample, only three of ten patients were diagnosed with MDD only. Investigating the costs for the different groups provided a more complete view on actual mental health services utilized and costs per patient. The population of our study sample demonstrated that the majority of patients with MDD have other psychiatric comorbidities that are associated with additional mental health service use and costs. Cost-of-illness studies that focus on the isolated costs of MDD only provide an overview of costs for a minority of patients with MDD in the real world.

At the time of MDD diagnosis, the mean age of patients in our study population was 44 years. Their age was not related 
Table 3 Patient characteristics, mental healthcare contacts, and treatment costs in patients with major depressive disorder with and without psychiatric comorbidities

Patients with MDD only

(1)

Patients with MDD
and other CM, without
PD (2)

PD (2)

11,090
17,235
59.7
$42.1 \pm 19$.
39.0

Subsequent treatment episodes, \%

Mental health service use per treatment episode, absolute numbers

Total, mean $\pm \mathrm{SD}$

Total, median

Inpatient days

Mean \pm SD

Median

In total episodes, \%

Day treatment days

$\begin{array}{ll}\text { Mean } \pm \text { SD } & 170 \pm 253 \\ \text { Median } & 102 \\ \text { In total episodes, \% } & 9.9\end{array}$

Psychiatric home care service visits

Mean + SD

Median

In total episodes, \%

Community-based daycare center visits

Mean \pm SD

Median

In total episodes, $\%$

Outpatient visits

Mean \pm SD

Median

In total episodes, \%

Total costs per treatment episode, $€$

Mean \pm SD

Median

Duration treatment episode, days

Mean \pm SD

Median
$20 \pm 40$

10

\section{$63 \pm 202$}

13

$150 \pm 377$

69

12.3

5.8

$195 \pm 290$

59

1.8

$23 \pm 41$

11

99.3

$11,612 \pm 49,534$

1508

$439 \pm 631$

233
$83 \pm 257$

17

$190 \pm 461$

76

14.5

$165 \pm 248$

91

12.2

$20 \pm 40$

10

7.6

$192 \pm 315$

56

2.6

$29 \pm 46$

14

99.4

$15,798 \pm 64,586$

1972

$492 \pm 647$

284
Patients with MDD and $\mathrm{PD}$, without other CM

(3)

3659

4812

62.6

$47.1 \pm 16.3$

23.1

Patients with MDD, $\quad p$-value $\mathrm{PD}$, and other CM

(4)

10,139

18,735

63.6

$<0.001^{*}$

$41.0 \pm 15.7<0.001^{*}$

54.

$<0.001^{*}$

$164 \pm 391$

$<0.001^{*}$

28

$233 \pm 483$

$202 \pm 480$

84

79

22.0

$242 \pm 349$

$222 \pm 322$

137

119

17.9

23.1

$40 \pm 88$

$41 \pm 84$

13

10.2

12.4

$162 \pm 265$

$219 \pm 306$

58

4.6

5.7

$41 \pm 63$

$47 \pm 82$

18

19

99.5

99.5

$23,933 \pm 77,271$

2900

$733 \pm 946$

370.5
$31,509 \pm 91,340$

3480

$709 \pm 895$

384

$C M$ comorbidities (psychiatric), $M D D$ major depressive disorder, $P D$ personality disorder, $S D$ standard deviation

*Significant $p$-value

to the age of first depressive episode but was related to the age of first treatment episode after MDD diagnosis in specialist mental healthcare. Patients are often treated by the GP or in primary mental healthcare before referral to specialist mental healthcare. Patients with comorbidities were younger, on average. This might be because of an increased tendency to seek help more quickly in people with MDD and psychiatric comorbidities than in people with MDD only [42]. 
Table 4 Relation between patient and clinical characteristics and mental healthcare use costs

\begin{tabular}{lllc}
\hline Characteristic & Coefficient, Exp(b) & Standard error & $95 \%$ CI \\
\hline Patient group (ref = MDD only) & & & \\
MDD, CM, no PD & 1.2319 & 0.0325 & $1.1699-1.2972$ \\
MDD, PD, no CM & 1.2745 & 0.0460 & $1.1875-1.3680$ \\
MDD, PD, CM & 1.7799 & 0.0491 & $1.6861-1.8789$ \\
Female sex (ref = male) & 1.0279 & 0.0193 & $0.9907-1.0665$ \\
Age (years) & 1.0055 & 0.0005 & $1.0045-1.0065$ \\
Year of start treatment episode (ref = 2000) & 1.0072 & 0.0028 & $0.001^{*}$ \\
Treatment episode recurrence (ref = first) & & & 0.144 \\
Subsequent & 0.7667 & $0.016-1.0128$ & $0.011^{*}$ \\
Treatment episode duration (days) & 1.0018 & 0.00002 & $0.7367-0.7979$ \\
Constant & 2020 & 90 & $1.0017-1.0018$ \\
\hline
\end{tabular}

$C I$ confidence interval, $C M$ comorbidities (psychiatric), $M D D$ major depressive disorder, $P D$ personality disorder

*Significant $p$-value

Table 5 Sensitivity analyses

\begin{tabular}{llll}
\hline & 3 months & 6 months & 9 months \\
\hline Duration treatment episode, days & & & \\
Mean \pm SD & $358 \pm 572$ & $579 \pm 780$ & $683 \pm 864$ \\
Median & 167 & 308 & 371 \\
Patients with subsequent treatment episodes, $\%$ & 51.2 & 35.0 & 28.4 \\
Costs of treatment episode, $€$ & & & \\
Mean \pm SD & $14,916 \pm 61,978$ & $21,186 \pm 74,192$ & $23,655 \pm 78,467$ \\
Median & 1160 & 2320 & 2784 \\
Gap between treatment episodes, days & & & \\
$\quad$ Mean \pm SD & $363 \pm 457$ & $641 \pm 554$ & $812 \pm 575$ \\
Median & 171 & 418 & 610 \\
\hline
\end{tabular}

$S D$ standard deviation

Compared with the rates of recurrence of depression episodes found in the literature [10], the proportion of subsequent treatment episodes in our study was relatively low $(35.0 \%)$. It is possible that a proportion of these patients had subsequent treatment outside the specialist mental healthcare setting. In addition, we only had a maximum of 12 years of follow-up time, with an average follow-up time of 1209 days.

GLM analysis showed that a subsequent treatment episode was associated with lower treatment costs. In a previous study, a recurrent depressive episode also predicted shorter durations of depressive episodes [41]. Presumably, patients with a recurrent depression better recognize the signals of the disorder and seek treatment more quickly. They might have also learned how to better cope with a depressive episode because of prior experiences. Furthermore, age and hospitalizations were significant cost drivers in other costof-illness studies [26, 29, 43], with hospitalizations being a major cost component. In our study, hospitalizations were also a major cost driver, contributing to $50 \%$ of total costs, though only occurring in $12.7 \%$ of the episodes.

The sensitivity analyses demonstrated that, in the episode definition of 3 months, almost $20 \%$ of the treatment episodes lasted less than 2 weeks. This could indicate that a 3-month gap is likely too short and not suitable in the definition of treatment episodes. In the 9-month definition, median duration and median costs of treatment episodes were more comparable to those of the 6-month definition, indicating that these definitions approached the actual treatment episode.

Our study sample was selected based on a primary MDD diagnosis. The additional costs in patients with comorbidities likely reflect longer treatment due to disease complexity or the use of combination treatment. Combination treatment is not always obvious, for instance when elements of cognitive behavioral treatment for depression can also affect personality aspects. Our findings showed that the combination of MDD and comorbid PD resulted in more mental 
health service use and costs than MDD and other psychiatric comorbidities. However, we cannot draw any conclusions with regard to the exact costs that were attributable to combination or more intensive treatment in case of comorbidities. Given the excess costs and care utilization, disease management programs should target both PD and MDD, maybe more than is currently done, for example, integrated treatment or combined treatment instead of several disorderspecific or consecutive treatments [44]. Conceptualizing and treating MDD as an isolated disorder may underestimate the prognosis of the majority of patients and the type of care that is appropriate [45].

Several strengths and limitations of this study should be considered. A major strength is that mental health service utilization and related costs were tracked over a long period of time. Data in the PCRNN contained actual utilized mental health services and clinical diagnoses, which are not based on self-report questionnaires. The latter are often prone to recall bias. Furthermore, in a relatively large sample, isolated episodes of care could be identified. This made it possible to compare the costs of treatment episodes of patients with MDD only and those with additional psychiatric comorbidities during the available timeframe. Although our study focused on PD, it would be interesting to compare other psychiatric comorbidities in future studies, given the available sample size.

A first limitation of the study is that information regarding the costs of medication usage was unavailable. However, we expect that medication use is relatively stable over the duration of an episode and would only be a minor part of the costs. The most prevalent antidepressants (fluoxetine, paroxetine, fluvoxamine, citalopram, sertraline, and paroxetine) cost less than $€ 1.20$ per day [46].

A second limitation was that the number of variables concerning patient characteristics (e.g., occupation, education) were unavailable. This is one disadvantage of using a large real-world observational dataset as opposed to a designed but smaller study cohort. In addition, it might be possible that a comorbidity is identified during a treatment episode. We expect that the impact of this limitation is small, as symptoms are more likely to be present at the start of the episode than to manifest during treatment.

A third limitation is that the data used in our study are somewhat outdated. The epidemiology of MDD remained relatively stable over time, and the available evidence-based treatments are mostly still valid in current treatment guidelines. Therefore, we believe that a better understanding of the mental health service utilization and costs of treatment and the role of psychiatric comorbidities on these remain relevant at this time.

Another limitation of our study is that we could not ascertain new-onset MDD diagnosis. MDD diagnoses prior to an individual's inclusion in the cohort were not available and might have been present, especially among individuals who were older in their cohort's first episode. This could impact our findings, as the number of past diagnoses might significantly affect treatment costs and service use. However, the 12-year follow-up time of our dataset partially addressed the problem of not being able to ascertain new-onset episodes. In addition, we distinguished between first treatment episodes for patients diagnosed with MDD with or without a history of specialist mental health service use for a non-MDD-related psychiatric diagnosis and subsequent treatment episodes in our cohort.

Finally, no information regarding reasons for treatment termination was available, so we do not know whether treatment episodes were terminated because of recovery or for other reasons. Patients in specialist mental healthcare might also be referred back to generalist mental health services when only residual symptoms are present. In such cases, a treatment episode does not end but continues in a less intensive mode. We performed sensitivity analyses to account for this lack of information.

\section{Conclusion}

The majority of patients with MDD in specialist mental healthcare have psychiatric comorbidities. Cost-of-illness studies that focus on MDD alone underestimate mental healthcare costs for most patients with MDD. This is especially true for a comorbid PD, which makes treatment longer and more costly. A better understanding of the costs of treatment episodes in specialist mental healthcare may help identify patients at risk of burdensome treatment trajectories, thereby providing the opportunity for future stratified disease management programs.

Supplementary Information The online version contains supplementary material available at https://doi.org/10.1007/s40273-021-01012-x.

Acknowledgements The authors thank Erwin Veermans, MSc, and Dr. Dennis Raven (both from RoQua, University Medical Center Groningen, Groningen, the Netherlands) for their efforts in setting up and coordinating the PCRNN database. We also thank all the mental healthcare organizations involved in the Psychiatric Case Register North Netherlands (GGZ Drenthe Mental Health Organization, GGZ Friesland Mental Health Organization, Lentis Psychiatric Institute, and Accare Child and Adolescent Psychiatry Center).

Code availability The syntax generated for the analyses is available from the corresponding author upon reasonable request.

\section{Declarations}

Funding This study was funded by Stichting De Friesland, Leeuwarden, the Netherlands (grant number DS29). Stichting De Friesland had no role in the study design; collection, analysis, and interpretation of the data; report writing; or the decision to submit the article for publication. 
Conflicts of interest Kan, Lokkerbol, Jörg, Visser, Schoevers, and Feenstra have no conflicts of interest that are directly relevant to the content of this article.

Ethics approval This research was conducted retrospectively from already available registry data. The Medical Ethics Review Board (METc) of the University Medical Center Groningen confirmed that no ethical approval was required.

Consent to Participate The METc of the University Medical Center Groningen granted exemption for the study, in line with the Dutch Medical Research involving Human Subjects Act.

Availability of Data Approval to analyze the data was obtained under the General Data Protection Regulation, provided that researchers would only access the data for specific research questions as agreed upon in the IMPROVE consortium. We are not legally or ethically allowed to publicly post our dataset.

Authors' contributions Conception and design: Kan, Jörg, Feenstra, and Lokkerbol. Kan, Lokkerbol, Feenstra, Jörg, and Visser analyzed the data. All authors interpreted the data. Kan, Jörg, and Feenstra drafted the article. All authors critically revised the article for important intellectual content and gave approval for the final version to be published.

Open Access This article is licensed under a Creative Commons Attribution-NonCommercial 4.0 International License, which permits any non-commercial use, sharing, adaptation, distribution and reproduction in any medium or format, as long as you give appropriate credit to the original author(s) and the source, provide a link to the Creative Commons licence, and indicate if changes were made. The images or other third party material in this article are included in the article's Creative Commons licence, unless indicated otherwise in a credit line to the material. If material is not included in the article's Creative Commons licence and your intended use is not permitted by statutory regulation or exceeds the permitted use, you will need to obtain permission directly from the copyright holder. To view a copy of this licence, visit http://creativecommons.org/licenses/by-nc/4.0/.

\section{References}

1. GBD 2017 Disease and Injury Incidence and Prevalence Collaborators. Global, regional, and national incidence, prevalence, and years lived with disability for 354 diseases and injuries for 195 countries and territories, 1990-2017: a systematic analysis for the Global Burden of Disease Study 2017. Lancet. 2018;392:1789-1858

2. de Graaf R, ten Have M, van Gool C, van Dorsselaer S. Prevalence of mental disorders and trends from 1996 to 2009. Results from the Netherlands Mental Health Survey and Incidence Study-2. Soc Psychiatry Psychiatr Epidemiol. 2012;47:203-213

3. Bromet E, Andrade LH, Hwang I, Sampson NA, Alonso J, de Girolamo G, de Graaf R, Demyttenaere K, Hu C, Iwata N, Karam AN, Kaur J, Kostyuchenko S, Lepine JP, Levinson D, Matschinger H, Mora ME, Browne MO, Posada-Villa J, Viana MC, Williams DR, Kessler RC. Cross-national epidemiology of DSM-IV major depressive episode. BMC Med. 2011;9:90-7015-9-90

4. Wells KB, Stewart A, Hays RD, Burnam MA, Rogers W, Daniels M, Berry S, Greenfield S, Ware J. The functioning and well-being of depressed patients: results from the Medical Outcomes Study. JAMA. 1989;262:914-9.
5. Broadhead WE, Blazer DG, George LK, Tse CK. Depression, disability days, and days lost from work in a prospective epidemiologic survey. JAMA. 1990;264:2524-8.

6. Murray CJ, Vos T, Lozano R, Naghavi M, Flaxman AD, Michaud C, et al. Disability-adjusted life years (DALYs) for 291 diseases and injuries in 21 regions, 1990-2010: a systematic analysis for the Global Burden of Disease Study 2010. Lancet. 2012;380:2197-223.

7. Global Burden of Disease Study 2013 Collaborators. Global, regional, and national incidence, prevalence, and years lived with disability for 301 acute and chronic diseases and injuries in 188 countries, 1990-2013: a systematic analysis for the Global Burden of Disease Study 2013. Lancet. 2015;386:743-800

8. Mathers CD, Loncar D. Projections of global mortality and burden of disease from 2002 to 2030. PLoS Med. 2006;3:e442.

9. Maj M, Veltro F, Pirozzi R, Lobrace S, Magliano L. Pattern of recurrence of illness after recovery from an episode of major depression: a prospective study. Am J Psychiatry. 1992; 149:795-800.

10. Hardeveld F, Spijker J, De Graaf R, Nolen WA, Beekman AT. Prevalence and predictors of recurrence of major depressive disorder in the adult population. Acta Psychiatr Scand. 2010;122:184-91.

11. Verhaak PF, Prins MA, Spreeuwenberg P, Draisma S, van Balkom TJ, Bensing JM, Laurant MG, van Marwijk HW, van der Meer K, Penninx BW. Receiving treatment for common mental disorders. Gen Hosp Psychiatry. 2009;31:46-55.

12. National Institute for Public Health and the Environment (Rijksinstituut voor Volksgezondheid en Milieu (RIVM)). In: Volksgezondheidenzorg.info - Cost of Illness database 2017. statline. rivm.nl. Accessed July 2020.

13. Kessler RC, Nelson CB, McGonagle KA, Liu J, Swartz M, Blazer DG. Comorbidity of DSM-III-R major depressive disorder in the general population: results from the US National Comorbidity Survey. Br J Psychiatry Suppl. 1996;30:17-30.

14. Kessler RC, Berglund P, Demler O, Jin R, Koretz D, Merikangas KR, Rush AJ, Walters EE, Wang PS, National Comorbidity Survey Replication. The epidemiology of major depressive disorder: results from the National Comorbidity Survey Replication (NCSR). JAMA. 2003;289:3095-3105

15. Keitner GI, Ryan CE, Miller IW, Kohn R, Epstein NB. 12-Month outcome of patients with major depression and comorbid psychiatric or medical illness (compound depression). Am J Psychiatry. 1991;148:345-50.

16. Friborg $\mathrm{O}$, Martinsen EW, Martinussen M, Kaiser S, Overgård $\mathrm{KT}$, Rosenvinge JH. Comorbidity of personality disorders in mood disorders: a meta-analytic review of 122 studies from 1988 to 2010. J Affect Disord. 2014;152-154:1-11.

17. Newton-Howes G, Tyrer P, Johnson T. Personality disorder and the outcome of depression: meta-analysis of published studies. $\mathrm{Br}$ J Psychiatry. 2006;188:13-20.

18. Newton-Howes G, Tyrer P, Johnson T, Mulder R, Kool S, Dekker $\mathrm{J}$, Schoevers R. Influence of personality on the outcome of treatment in depression: systematic review and meta-analysis. J Pers Disord. 2014;28:577-93.

19. Patience DA, McGuire RJ, Scott AI, Freeman CP. The Edinburgh Primary Care Depression Study: personality disorder and outcome. Br J Psychiatry. 1995;167:324-30.

20. De Bolle M, De Fruyt F, Quilty LC, Rolland JP, Decuyper M, Bagby RM. Does personality disorder co-morbidity impact treatment outcome for patients with major depression? A multi-level analysis. J Pers Disord. 2011;25:1-15.

21. Koppers D, Kool M, Van H, Driessen E, Peen J, Dekker J. The effect of comorbid personality disorder on depression outcome after short-term psychotherapy in a randomised clinical trial. BJPsych Open. 2019;5:e61. 
22. Bender DS, Dolan RT, Skodol AE, Sanislow CA, Dyck IR, McGlashan TH, Shea MT, Zanarini MC, Oldham JM, Gunderson JG. Treatment utilization by patients with personality disorders. Am J Psychiatry. 2001;158:295-302.

23. Wiegand HF, Godemann F. Increased treatment complexity for major depressive disorder for inpatients with comorbid personality disorder. Psychiatr Serv. 2017;68:524-7.

24. Black DW, Bell S, Hulbert J, Nasrallah A. The importance of Axis II in patients with major depression. A controlled study J Affect Disord. 1988;14:115-22.

25. Greenberg PE, Kessler RC, Birnbaum HG, Leong SA, Lowe SW, Berglund PA, Corey-Lisle PK. The economic burden of depression in the United States: how did it change between 1990 and 2000? J Clin Psychiatry. 2003;64:1465-75.

26. Tomonaga Y, Haettenschwiler J, Hatzinger M, Holsboer-Trachsler E, Rufer M, Hepp U, Szucs TD. The economic burden of depression in Switzerland. Pharmacoeconomics. 2013;31:237-50.

27. Luppa M, Heinrich S, Angermeyer MC, König H, Riedel-Heller SG. Cost-of-illness studies of depression: a systematic review. J Affect Disord. 2007;98:29-43.

28. Thomas CM, Morris S. Cost of depression among adults in England in 2000. Br J Psychiatry. 2003;183:514-9.

29. Kleine-Budde K, Muller R, Kawohl W, Bramesfeld A, Moock J, Rossler W. The cost of depression - a cost analysis from a large database. J Affect Disord. 2013;147:137-43.

30. Konig H, Konig HH, Konnopka A. The excess costs of depression: a systematic review and meta-analysis. Epidemiol Psychiatr Sci. 2019;29:e30.

31. Simon G, Ormel J, VonKorff M, Barlow W. Health care costs associated with depressive and anxiety disorders in primary care. Am J Psychiatry. 1995; 152:352.

32. Bosmans JE, de Bruijne MC, de Boer MR, van Hout H, van Steenwijk P, van Tulder MW. Health care costs of depression in primary care patients in The Netherlands. Fam Pract. 2010;27:542-8.

33. Le TK, Curtis B, Kahle-Wrobleski K, Johnston J, Haldane D, Melfi C. Treatment patterns and resource use among patients with comorbid diabetes mellitus and major depressive disorder. J Med Econ. 2011;14:440-7.

34. Mausbach BT, Bos T, Irwin SA. Mental health treatment dose and annual healthcare costs in patients with cancer and major depressive disorder. Health Psychol. 2018;37:1035-40.

35. Wagner CJ, Metzger FG, Sievers C, Marschall U, L'hoest H, Stollenwerk B, Stock S. Depression-related treatment and costs in Germany: Do they change with comorbidity? A claims data analysis. J Affect Disord. 2016;193:257-266

36. Cuijpers P, Smit F, Penninx BW, de Graaf R, ten Have M, Beekman AT. Economic costs of neuroticism: a population-based study. Arch Gen Psychiatry. 2010;67:1086-93.

37. American Psychiatric Association. Diagnostic and Statistical Manual of Mental Disorders (DSM-IV). 4th edn. Washington DC;1994

38. Hakkaart-van Roijen L, Van der Linden N, Bouwmans C, Kanters T, Tan SS. Kostenhandleiding: Methodologie van kostenonderzoek en referentieprijzen voor economische evaluaties in de gezondheidszorg. National Health Care Institute. 2015

39. Gregori D, Petrinco M, Bo S, Desideri A, Merletti F, Pagano E. Regression models for analyzing costs and their determinants in health care: an introductory review. Int J Qual Health Care. 2011;23:331-41.

40. Keller MB, Shapiro RW, Lavori PW, Wolfe N. Recovery in major depressive disorder: analysis with the life table and regression models. Arch Gen Psychiatry. 1982;39:905-10.

41. Spijker J, de Graaf R, Bijl RV, Beekman AT, Ormel J, Nolen WA. Duration of major depressive episodes in the general population: results from The Netherlands Mental Health Survey and Incidence Study (NEMESIS). Br J Psychiatry. 2002;181:208-13.

42. Raven D, Jörg F, Visser E, Oldehinkel AJ, Schoevers RA. Timeto-treatment of mental disorders in a community sample of Dutch adolescents. A TRAILS study. Epidemiol Psychiatr Sci. 2017;26:177-88.

43. Berto P, D'Ilario D, Ruffo P, Di Virgilio R, Rizzo F. Depression: cost-of-illness studies in the international literature, a review. J Ment Health Policy Econ. 2000;3:3-10.

44. Van H, Kool M. Integrated treatment for patients with comorbid depression and personality disorders. Curr Opin Psychiatry. 2020;33:70-5.

45. Verduijn J, Verhoeven JE, Milaneschi Y, Schoevers RA, van Hemert AM, Beekman ATF, Penninx BWJH. Reconsidering the prognosis of major depressive disorder across diagnostic boundaries: full recovery is the exception rather than the rule. BMC Med. 2017; 15:215-017-0972-8

46. National Health Care Institute. In: Medicijnkosten.nl. https://www. medicijnkosten.nl. Accessed June, 112020.

\section{Authors and Affiliations}

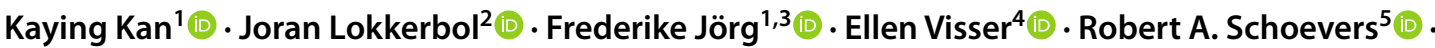 Talitha L. Feenstra ${ }^{6,7}$ (i)}

1 University of Groningen, University Medical Center Groningen, University Center for Psychiatry, Rob Giel Research Center, Interdisciplinary Centre for Psychopathology and Emotion Regulation, PO Box 30001, Hospital zip code CC72, 9700 RB Groningen, The Netherlands

2 Centre of Economic Evaluation and Machine Learning, Trimbos Institute (Netherlands Institute of Mental Health and Addiction), Utrecht, The Netherlands

3 GGZ Friesland, Research Department, Leeuwarden, The Netherlands
4 University of Groningen, University Medical Center Groningen, University Center for Psychiatry, Rob Giel Research Center, Groningen, The Netherlands

5 University of Groningen, University Medical Center Groningen, University Center for Psychiatry, Interdisciplinary Centre for Psychopathology and Emotion Regulation, Groningen, The Netherlands

6 University of Groningen, Department of Science and Engineering, Groningen Research Institute of Pharmacy, Groningen, The Netherlands

7 National Institute for Public Health and the Environment (RIVM), Bilthoven, The Netherlands 